understood that incentivising enables beneficial change in doctor's fill rates, highlighted in HEE's 'Training in Smaller Places' report.

Evidence for long-term impact of enhanced recruitment is maturing; opinions remain divided. Providing financial incentive where training schemes are specifically targeted to promote retention of future medical workforce is novel and we are the only UK integrated healthcare system using TERS on a regional footprint for all specialties.

In 2018, HEE NE provided a TERS payment of $£ 7000$ for trainees taking up a 12-month Specialty Training or Foundation Post in North Cumbria. Since 2019, the Local Education Provider has continued the scheme.

Post Fill rates TERS resulted in a $20 \%$ increase in fill rate across training grades. The lower 2018/19 FY2 fill rate was a result of the low FY1 fill rate in 2017/18 before the introduction of TERS.

Application Score TERS has dramatically improved the average Foundation Programme (FP) application score since 2018, from 71.6 to 77.5 in 2020. During the same time period the average score for the North East has gone from 79.2 to 80 .

Surveys $100 \%$ reported TERS positively influenced their decision making, 42\% ranked Cumbria higher in selection rankings, $74 \%$ that $£ 7000$ was the right amount and $63 \%$ felt TERS positively influenced their decision to stay in Cumbria.

Financial benefits TERS has cost $£ 941,597$ to date in direct expenditure however we anticipate it has had improvements in overall locum spend, requirement for Trust grade positions and excess hours fines, figures we are looking to finalise imminently.

Conclusion TERS attracts more doctors of a higher calibre to Cumbria, benefiting our services and reducing disadvantage to our population. We plan to continue the scheme and further analyse its impact.

\section{THE INFLUENCE OF LEADERSHIP DEVELOPMENT AND PSYCHOLOGICAL CAPITAL ON BURNOUT AND TURNOVER}

Michelle Jacobs. Liberty University, USA

\subsection{6/leader-2021-FMLM.65}

Background Organizations should strive to function at the highest level possible. One way to foster organizational success is through leadership development (LD), though such education for middle managers was limited. Additionally, new midlevel managers in healthcare experienced decreased wellbeing. These issues are both harmful to institutional outcomes.

Aim The aim was to explore the impact of LD and psychological capital (PsyCap) on new middle managers' burnout and turnover intention.

Methods This qualitative, single-case study occurred in the Mid-West United States. Three data collection techniques were utilized, with the primary method being semi-structured interviews of 19 new middle managers. Data was also collected by observing LD activities and reviewing organizational documents about existing LD programs.

Results Participants acknowledged a high prevalence of burnout and turnover intention. Subjects claimed to experience burnout related to the organization's LD activities. The PsyCap constructs helped decrease burnout and turnover. Managers with high levels of self-efficacy and resiliency, though, expressed increased incidents of burnout. The combination of effective LD and positive PsyCap appeared to have a compounding effect of decreasing burnout and turnover.

Conclusion Healthcare organizations should utilize the data identified from this study to decrease and prevent burnout and turnover among their leaders. Administrators are encouraged to improve the effectiveness of their LD programs and employ managers with positive PsyCap elements. The adverse impact of self-efficacy and resiliency on burnout was contrary to the limited existing literature and should be examined further. Additionally, a combined exploration of LD and PsyCap related to burnout had not been studied, resulting in a unique contribution of this investigation; more research is necessary.

\section{THE INTRODUCTION OF A CRITICAL CARE HANDOVER AND SAFETY CHECKLIST}

Kayur Patel, Matthew Willcocks. Queen Victoria Hospital, East Grinstead, UK

\subsection{6/leader-2021-FMLM.66}

Aim Our hospital has a small six-bedded critical care unit (CCU) dealing with complex post-surgical patients. Each handover took place verbally without structure before our intervention. To improve patient safety and communication, a structured handover was sort.

Method A quality improvement methodology was used to produce iterative designs of a handover board. Initial ideas focused on a computer-based handover, but a more visual solution was requested.

Results A questionnaire was performed pre-and post-intervention. The results demonstrated significant improvements in all aspects of handover. Initially, 29\% of respondents knew the names of nurse-in-charge, registrar or consultant for a shift. This improved to $70 \%$ post-intervention. Due to the pandemic, changes in handover arrangement meant $29 \%$ did not know the location of handover. This increased to $70 \%$ following our project. Only 33\% knew the number of discharges and 50\% knew expected admissions for a shift. Both increased to over $70 \%$ after the intervention. Preintervention only $39 \%$ knew if they had bed capacity and staffing on a particular day, this increased to $70 \%$ after implementation.

Discussion A laminated handover board now enables a quick glance for all staff about the status of CCU. A co-introduced safety, 'Handover checklist' ensures critical issues are highlighted and handover content is consistent. This is especially useful at our institution where surgery involving difficult airways occur frequently.

Stakeholder feedback was sought throughout to ensure sustainability and effectiveness. During implementation, care was taken to ensure the current challenges and aims of the project were understood by participants. This certainly helped to conquer barriers to adoption.

Conclusion Although a simple, we have improved the safety of handover in our CCU. Its implementation has also seen closer communication between doctors and nurses. As a referral centre, it has made accepting patients more streamlined. 